\title{
Anomalous surface brightness fluctuations in NGC 4489^
}

\author{
S. Mei ${ }^{1,2}$, D. R. Silva ${ }^{2, \star \star}$, and P. J. Quinn ${ }^{2}$ \\ 1 Observatoire Midi-Pyrénées, 14 Av. E. Belin, 31400 Toulouse, France \\ ${ }^{2}$ European Southern Observatory, Karl-Schwarzschild-Strasse 2, 85748 Garching, Germany
}

Received 18 February 2000 / Accepted 30 October 2000

\begin{abstract}
Anomalously high $K$-band surface brightness fluctuations (SBF) have been reported in NGC 4489 by Pahre \& Mould (1994), Jensen et al. (1996) and Jensen et al. (1998). However, these conclusions were uncertain because of relatively low signal-to-noise data. New high signal-to-noise data for NGC 4489 have been obtained at the NOAO/KPNO $2.1 \mathrm{~m}$ and the ESO/La Silla $3.5 \mathrm{~m}$ NTT telescopes. Adopting the $I$-band SBF distance modulus determined by Tonry et al. (2000b) and the $\bar{M}_{I}$ versus $(V-I)$ calibration of Tonry et al. (2000a), a value of $\bar{M}_{K}=-6.29 \pm 0.10 \mathrm{mag}$ was derived. Relative to the average empirical $\bar{M}_{K}$ derived for giant ellipticals by Jensen et al. (1998) $\left(\bar{M}_{K}=-5.61 \pm 0.12 \mathrm{mag}\right)$, the detection of an anomalous $K$-band SBF in NGC 4489 is confirmed at the two sigma level. Such anomalous fluctuations could be caused by an extended giant branch, consisting of either intermediate-age AGB stars above the tip of the first-ascent giant branch or high-metallicity first-ascent giants, or by lack of a full understanding of the $K$-band SBF calibration. This result raises questions about the accuracy of $K$-band SBF distance measurements for elliptical galaxies with unknown stellar composition and underscores the need for $\bar{M}_{K}$ measurements over a larger range of color and luminosity.
\end{abstract}

Key words. galaxies: distances and redshift - individual: NGC 4489

\section{Introduction}

The Poissonian statistics of stellar populations in each pixel of a galaxy image produces fluctuations. While the mean flux per pixel does not depend on distance, the variance of these fluctuations is inversely proportional to the square of the galaxy distance. When properly calibrated this variance can then be used as a distance indicator. Surface Brightness Fluctuations (SBF) are defined as the variance of the flux of the fluctuations normalized to the mean flux of the galaxy in each pixel Tonry \& Schneider 1988. The stars that contribute the most to the SBF are then the brightest stars in the intrinsic luminosity function, typically red giants in old stellar populations (a recent review on the method has been given by Blakeslee et al. 1999).

$I$-band SBF have been used successfully to measure elliptical and S0 distances up to $4000 \mathrm{~km} \mathrm{~s}^{-1}$ from ground

Send offprint requests to: S. Mei, e-mail: mei@ast.obs-mip.fr * Based on observations performed at the European Southern Observatory, La Silla, Chile; ESO program No. 62.N-0876.

** Visiting Astronomer, Kitt Peak National Observatory, National Optical Astronomical Observatories, operated by the Association of Universities for Research in Astronomy, Inc., under cooperative agreement with the National Science Foundation. based telescopes and $7000 \mathrm{~km} \mathrm{~s}^{-1}$ on the Hubble Space Telescope (HST) (Sodemann \& Thomsen 1995, 1996; Ajhar et al. 1997; Tonry et al. 1997; Thomsen 1997; Lauer et al. 1998; Pahre et al. 1999; Blakeslee et al. 1999; Tonry et al. 2000a, 2000b). Recently SBF measurements have been attempted in the infrared $K$-band for several reasons (Luppino \& Tonry 1993; Pahre \& Mould 1994; Jensen et al. 1996, 1998, 1999).

First of all the contrast between brightest red giants and the underlying stellar populations is more extreme in the $K$-band, producing larger SBF at any given distance. Second, at ground facilities, $K$-band seeing is intrinsically better than $I$-band seeing which also enhances the SBF contrast. Moreover the color contrast between $K$-band SBF and external sources (globular clusters and background galaxies) is larger than in the $I$-band. Finally both empirical and theoretical studies suggest that absolute $K-$ band SBF is almost constant with age for solar-metallicity stellar populations (Pahre \& Mould 1994; Jensen et al. 1996, 1998, 1999; Mei et al. 1999; Liu et al. 2000; Mei et al. 2000).

These arguments suggest that in the infrared it should be possible to determine SBF distances for galaxies at larger distances than at optical wavelengths (Jensen et al. 1998). However, the $K$-band has one major disadvantage for ground-based studies - the background is significantly brighter making it much more difficult to detect and 
correct for globular cluster and background galaxy contamination and increasing the background shot noise.

Initial $K$-band SBF studies suggested that the $\bar{M}_{K}$ varied very little from galaxy-to-galaxy (Pahre \& Mould 1994; Jensen et al. 1996). However, these studies also argued that two Virgo ellipticals (NGC 4365 and NGC 4489) had significantly brighter than average $\bar{M}_{K}$ values suggesting the presence of an extended giant branch. This conclusion, however, was weakened by two problems: (1) no correction was made for globular cluster and background galaxy contamination; and (2) the SBF measurements were based on relatively low signal-to-noise data. Jensen et al. (1998) re-measured $\bar{M}_{K}$ for NGC 4365, using $I$-band data to determine a correction for globular cluster contamination, and concluded that NGC 4365 was a member of the background $\mathrm{W}$ cloud and the $\bar{M}_{K}$ for this galaxy was consistent with other Virgo ellipticals. In short, NGC 4365 appears to have a normal giant branch. No additional study of NGC 4489 has been published until now.

In this study, new observations with significantly higher signal-to-noise and a new SBF analysis for NGC 4489 are presented. The observations are described in Sect. 2, the SBF analysis in Sect. 3. The results of this analysis are presented in Sects. 4 and 5 .

\section{Observations}

\subsection{NGC 3379 and NGC 4489}

The galaxies NGC 3379 in the Leo cluster and NGC 4489 in Virgo were observed. NGC 3379 has very wellestablished near-IR photometry (Frogel et al. 1978; Silva \& Bothun 1998) and has been already deeply studied in the $I$-band by Sodemann \& Thomsen (1995). It has been used here as a fiducial galaxy. NGC 4489 is a low luminosity dwarf elliptical galaxy. Its position angle and ellipticity vary in a significant way with radius. Its profile deviates from a De Vaucouleurs law, rising steeply towards the center. It has a $\mathrm{Mg}_{2}$ index of 0.198 , different from the other Virgo galaxies.

\subsection{Observations}

Data for NGC 3379 and NGC 4489 were obtained during two different observing runs. The first dataset was obtained at the Kitt Peak National Observatory $2.1 \mathrm{~m}$ on 7 February 1999. The imaging camera was ONIS (OSU-NOAO Infrared Imaging Spectrometer) with a InSb $512 \times 1024$ array detector. The gain is $4 \mathrm{e}^{-} / \mathrm{ADU}$, the read out noise $\approx 9 \mathrm{ADU}$, the dark current $\approx 1.2 \mathrm{e}^{-} \mathrm{s}^{-1}$. The pixel scale was $0.34^{\prime \prime}$ and the field of view $6^{\prime} \times 3^{\prime}$. The filter that was used was a $K$ short filter $K^{\mathrm{sh}}$, centered at $2.16 \mu \mathrm{m}$. The $K^{\text {sh }}$ filter is centered at shorter wavelength than the standard $K$ filter to reduce thermal background and at larger wavelength than the $K^{\prime}$ filter (Wainscoat \& Cowie 1992). Pahre \& Mould (1994) estimated the difference between this filter and the standard $K$-band is of the order of 0.02 magnitudes for elliptical galaxies. We do not scale our results to the standard $K$-band filter, meaning the calculated $K$-band SBF magnitudes are magnitudes in the $K^{\text {sh }}$ filter.

The seeing was $1^{\prime \prime}$ for NGC 3379 and $0.9^{\prime \prime}$ for NGC 4489. The photometric zero-point and the extinction coefficient were extracted from the observations of NICMOS red standards (Persson et al. 1998). A photometric zero-point $m_{1}=21.32 \mathrm{mag}$ and an extinction coefficient of $0.1 \mathrm{mag}$ in the $K^{\mathrm{sh}}$ were determined. The sky brightness was on average 12.7 mag $\operatorname{arcsec}^{-2}$. NGC 3379 was observed during a non-photometric period.

Each galaxy was observed in a sequence of backgroundgalaxy-background sets. At each position, the integration time was $10 \times 6 \mathrm{~s}$. After each set, a small random dither was executed to facilitate removal of bad pixels. The total exposure times were $360 \mathrm{~s}$ for NGC 3379 and $1700 \mathrm{~s}$ for NGC 4489.

The second dataset was obtained using the $3.5 \mathrm{~m}$ New Technology Telescope (NTT) at the European Southern Observatory, La Silla, Chile, on 26 March 1999. The instrument used was SOFI (Son OF ISAAC) with a Hawaii $\mathrm{HgCdTe} 1024 \times 1024$ detector array. The gain is $5.53 \mathrm{e}^{-} / \mathrm{ADU}$, the read out noise $2.1 \mathrm{ADU}$, the dark current $<0.1 \mathrm{e}^{-} \mathrm{s}^{-1}$. The pixel scale was $0.145^{\prime \prime} /$ pixel, with a field of view $2.47^{\prime} \times 2.47^{\prime}$. The filter was again a $K^{\text {sh }}$ filter. The seeing was $0.75^{\prime \prime}$ for NGC 3379 and $0.85^{\prime \prime}$ for NGC 4489. A photometric zero-point $m_{1}=22.32 \mathrm{mag}$ and an extinction coefficient of $0.07 \mathrm{mag}$ were determined from NICMOS standards with solar-type colors (Persson et al. 1998). The sky brightness was on average $12.6 \mathrm{mag} \operatorname{arcsec}^{-2}$. Both galaxies were observed under photometric conditions.

NGC 3379 was observed in a sequence of galaxybackground sets. At each position, the integration time was $4 \times 15 \mathrm{~s}$. NGC 4489 was observed in a sequence of background-galaxy-background sets. At each position, the integration time was $3 \times 20 \mathrm{~s}$. After each set, a small random dither was executed to facilitate removal of bad pixels. The total exposure times were $1380 \mathrm{~s}$ for NGC 3379 and $3780 \mathrm{~s}$ for NGC 4489.

The two sets of data were similarly processed. Each galaxy image was background subtracted and then divided by a normalized dome flat field. Dark current was subtracted by this operation. Bad pixels and cosmic rays were eliminated by a sigma clipping algorithm while combining the images using the IRAF task IMCOMBINE. Subpixel registration was not used to avoid the introduction of correlated noise between the pixels into the images.

\section{Surface brightness fluctuations analysis}

Both datasets were analyzed by the SBF extraction standard technique used e.g. by Tonry \& Schneider (1988). A smooth galaxy model was built by fitting the galaxy isophotes and then subtracted from the image. Visible external sources were not included in the fitting procedure. External point sources were then identified using 
Table 1. SBF measurements for various annuli of NGC 3379 from NTT observations

\begin{tabular}{|c|c|c|c|c|c|c|c|}
\hline Annulus & $P_{0}$ & $\sigma_{P_{0}}$ & $P_{0} / P_{1}$ & $P_{\mathrm{es}}$ & $\bar{m}_{K}$ & $\sigma_{\bar{m}_{K}}$ & $m_{\text {cut }}$ \\
\hline$\left(^{\prime \prime}\right)$ & $\left(\mathrm{ADU} \mathrm{s}^{-1}\right)$ & $\left(\mathrm{ADU} \mathrm{s}^{-1}\right)$ & & $\left(\mathrm{ADU} \mathrm{s}^{-1}\right)$ & $(\mathrm{mag})$ & $(\mathrm{mag})$ & $(\mathrm{mag})$ \\
\hline \hline $12-16$ & 0.085 & 0.007 & 25 & 0.0076 & 24.86 & 0.1 & 19 \\
\hline $16-21$ & 0.096 & 0.005 & 20 & 0.0076 & 24.71 & 0.07 & 19 \\
\hline $21-26$ & 0.094 & 0.003 & 15 & 0.0076 & 24.69 & 0.04 & 19 \\
\hline $26-30$ & 0.100 & 0.002 & 13 & 0.0076 & 24.66 & 0.04 & 19 \\
\hline $30-35$ & 0.100 & 0.001 & 9 & 0.0076 & 24.66 & 0.03 & 19 \\
\hline $35-39$ & 0.094 & 0.002 & 8 & 0.0076 & 24.73 & 0.04 & 19 \\
\hline $2-39$ & 0.093 & 0.003 & 13.5 & 0.0076 & 24.70 & 0.03 & 19 \\
\hline \hline Mean & & & - & - & 24.72 & 0.06 & - \\
\hline
\end{tabular}

Table 2. SBF measurements for various annuli of NGC 4489 from KPNO observations

\begin{tabular}{|c|c|c|c|c|c|c|c|}
\hline Annulus & $P_{0}$ & $\sigma_{P_{0}}$ & $P_{0} / P_{1}$ & $P_{\mathrm{es}}$ & $\bar{m}_{K}$ & $\sigma_{\bar{m}_{K}}$ & $m_{\text {cut }}$ \\
\hline$\left(^{\prime \prime}\right)$ & $\left(\mathrm{ADU} \mathrm{s}^{-1}\right)$ & $\left(\mathrm{ADU} \mathrm{s}^{-1}\right)$ & & $\left(\mathrm{ADU} \mathrm{s}^{-1}\right)$ & $(\mathrm{mag})$ & $(\mathrm{mag})$ & $(\mathrm{mag})$ \\
\hline \hline $2-7$ & 0.032 & 0.005 & 10 & 0.0009 & 24.85 & 0.15 & 19.9 \\
\hline $7-12$ & 0.026 & 0.004 & 4 & 0.0009 & 25.08 & 0.14 & 19.9 \\
\hline $12-16$ & 0.030 & 0.005 & 3 & 0.0009 & 24.92 & 0.13 & 19.9 \\
\hline $2-16$ & 0.026 & 0.001 & 3 & 0.0009 & 25.08 & 0.03 & 19.9 \\
\hline \hline Mean & & & - & - & 24.98 & 0.06 & - \\
\hline
\end{tabular}

Table 3. SBF measurements for various annuli of NGC 4489 from NTT observations

\begin{tabular}{|c|c|c|c|c|c|c|c|}
\hline Annulus & $P_{0}$ & $\sigma_{P_{0}}$ & $P_{0} / P_{1}$ & $P_{\mathrm{es}}$ & $\bar{m}_{K}$ & $\sigma_{\bar{m}_{K}}$ & $m_{\text {cut }}$ \\
\hline$\left(^{\prime \prime}\right)$ & $\left(\mathrm{ADU} \mathrm{s}^{-1}\right)$ & $\left(\mathrm{ADU} \mathrm{s}^{-1}\right)$ & & $\left(\mathrm{ADU} \mathrm{s}^{-1}\right)$ & $(\mathrm{mag})$ & $(\mathrm{mag})$ & $(\mathrm{mag})$ \\
\hline \hline $2-7$ & 0.074 & 0.01 & 50 & 0.0022 & 24.96 & 0.18 & 19.9 \\
\hline $7-12$ & 0.068 & 0.007 & 40 & 0.0022 & 25.04 & 0.11 & 19.9 \\
\hline $12-16$ & 0.076 & 0.008 & 25 & 0.0022 & 24.91 & 0.13 & 19.9 \\
\hline $2-16$ & 0.070 & 0.002 & 25 & 0.0022 & 25.00 & 0.03 & 19.9 \\
\hline \hline Mean & & & - & - & 24.97 & 0.06 & - \\
\hline
\end{tabular}

Sextractor (Bertin \& Arnouts 1996) and subtracted. The residual image was smoothed on a scale ten times the width of the PSF. This smoothed image was subtracted from the residual image to correct residual sky-subtraction errors. That image was divided by the square root of the galaxy model to make the amplitude of the SBF fluctuations constant across the entire image. Finally, a point spread function (PSF) profile was determined from the bright stars in the image and normalized at $1 \mathrm{ADUs}^{-1}$.

Various annuli were then analysed. The photometric completeness function was calculated in each annulus by adding simulated point source images to the original, galaxy subtracted image. The photometric completeness was calculated in intervals of $0.1 \mathrm{mag}$, as the ratio of the simulated and the extracted point sources. The external point sources were masked up to a cut-off magnitude $m_{\text {cut }}$, whose value for each annulus is given in Table 2, for KPNO observations, and in Tables 1 and 3 for NTT observations. Next, the image power spectrum was calculated in each annulus and normalized to the number of non-zero points in the annulus. The power spectrum was azimuthally averaged.

The total image power spectrum is the sum of two components: a constant power spectrum due to the white noise, $P_{1}$ and a power spectrum of the fluctuation and point sources. Both components are convolved by the PSF in the spatial domain. In the Fourier domain this second component is given by a constant $P_{0}$ multiplied by the power spectrum of the PSF:

$E_{\text {gal }}=P_{0} E_{\mathrm{PSF}}+P_{1}$

To calculate $P_{0}$ and $P_{1}$, a robust linear least square fit, minimizing absolute deviation (Numerical Recipes, Press et al. 1992), was made to the power spectra of each 


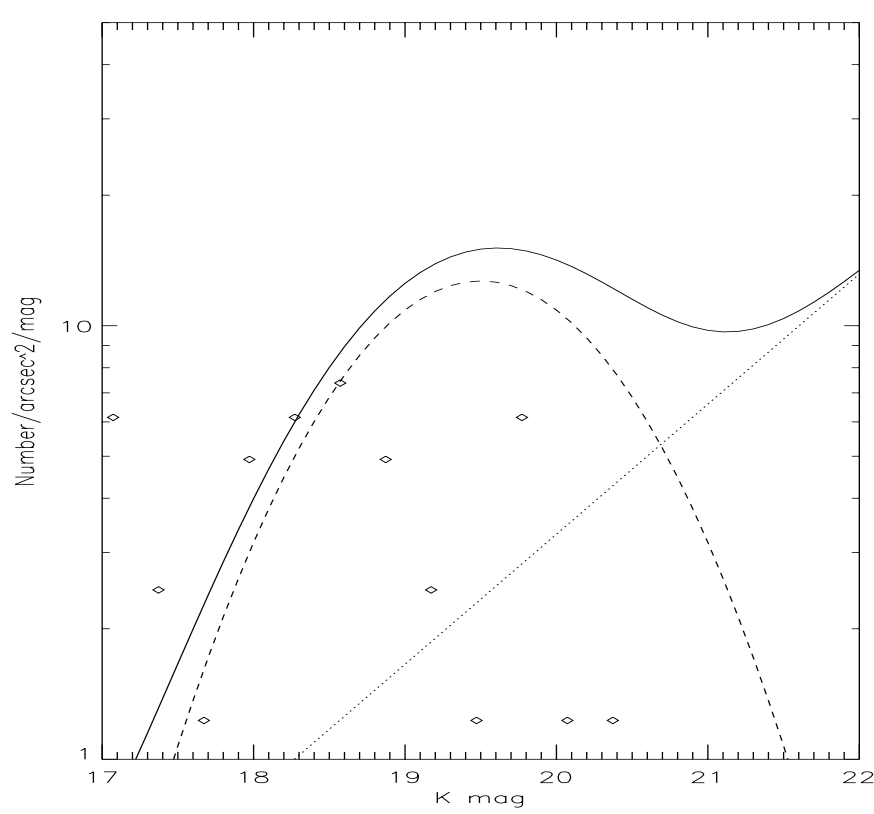

Fig. 1. NGC 3379 external source luminosity function derived from the NTT data. The continuous line shows the sum of the adopted globular cluster plus background galaxy luminosity functions. The dashed line shows the globular cluster luminosity function, while the dotted line shows the background galaxy luminosity function. In total, 38 sources were detected between $\approx 15^{\prime \prime}$ and $\approx 60^{\prime \prime}$

annuli. Low (typically less than 4) wave number points were excluded from the fit since they are contaminated by the galaxy subtraction and subsequent smoothing errors, plus residual background variance contribution, as pointed out e.g. by Jensen et al. (1999).

$P_{0}$ was then corrected for the contribution of point source fluctuations $P_{\mathrm{es}}$, estimated from the equations:

$P_{\mathrm{es}}=\sigma_{\mathrm{gc}}^{2}+\sigma_{\mathrm{bg}}^{2}$

as described by Blakeslee \& Tonry (1995): $\sigma_{\mathrm{gc}}^{2}$ is the contribution to the fluctuations given by globular clusters, $\sigma_{\mathrm{bg}}^{2}$ is the contributions by background galaxies. It is assumed that the luminosity function of the globular clusters is given by:

$N_{\text {gc }}(m)=\frac{N_{\text {ogc }}}{\sqrt{2 \pi} \sigma} \mathrm{e}^{\frac{-\left(m-m_{\text {peak }}\right)^{2}}{2 \sigma^{2}}}$,

with $\sigma=1.35, M_{\text {peak } V}=-7.5$ (Ferrarese et al. 2000) and $(V-K)=2.23$ for NGC 4489 and $m_{\text {peak } B}=22.70$ and $\sigma=0.9$ from Pritcher \& Van den Bergh (1985) for NGC 3379 and standard $(V-K)$ color $(\approx 3)$ from KisslerPatig (2000). We have verified that exact details in the adopted globular cluster and background galaxy luminosity functions have little effect on the final measurement of SBF amplitudes, as pointed out i.e. from Blakeslee et al. (1999a). For the background galaxies a power-law luminosity function was assumed:

$N_{\text {bg }}(m)=N_{\text {obg }} 10^{\gamma m}$

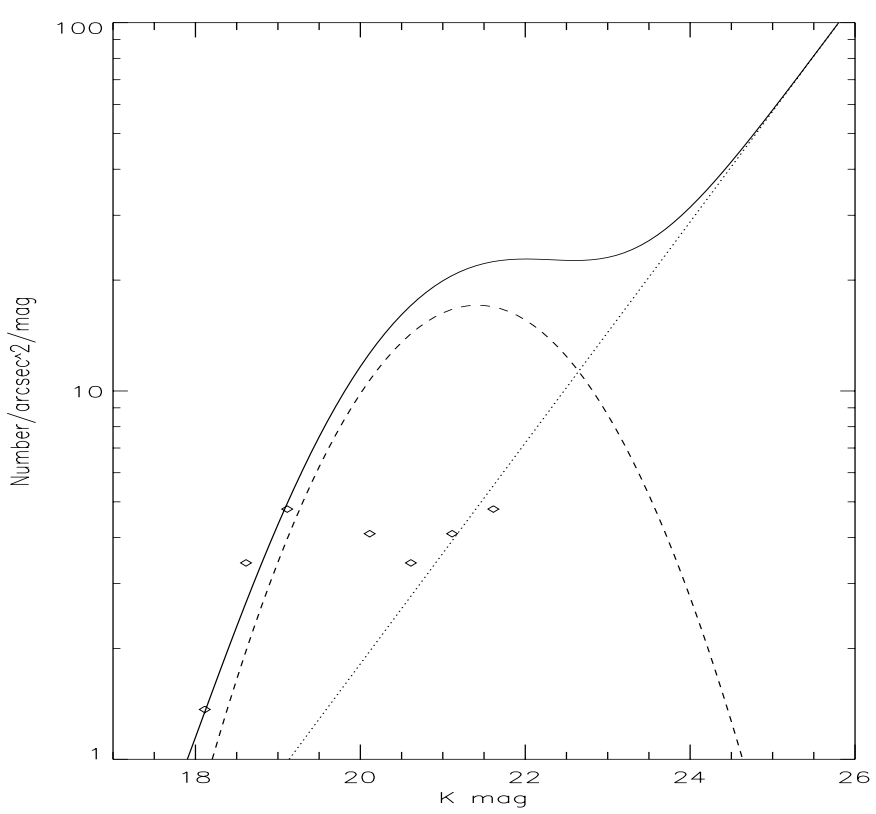

Fig. 2. NGC 4489 external source luminosity function derived from the NTT data. The continuous line shows the sum of the adopted globular cluster plus background galaxy luminosity functions. The dashed line shows the globular cluster luminosity function, while the dotted line shows the background galaxy luminosity function. In total, 62 sources were detected between $\approx 2^{\prime \prime}$ and $\approx 60^{\prime \prime}$

with $\gamma=0.3$ (Cowie et al. 1994). $N_{\text {ogc }}$ and $N_{\text {obg }}$ were estimated by fitting these equations to the observed composite luminosity function determined from the external sources extracted from the image. The actual $m_{\text {cut }}$ used are given in Tables $1-3$, while the observed external source luminosity functions for NGC 3379 and NGC 4489 are shown respectively in Figs. 1 and 2, as derived from the NTT dataset. The fit has been made as from the sum of the globular cluster plus background galaxies luminosity function:

$$
\begin{aligned}
N(m) & =N_{\mathrm{gc}}(m)+N_{\mathrm{bg}}(m) \\
& =\frac{N_{\text {ogc }}}{\sqrt{2 \pi} \sigma} \mathrm{e}^{\frac{-\left(m-m_{\text {peak }}\right)^{2}}{2 \sigma^{2}}}+N_{\text {obg }} 10^{\gamma m} .
\end{aligned}
$$

We keep all the parameters fixed in the fit, but $N_{\text {ogc }}$ and $N_{\text {obg. }}$. We have assumed a galaxy distance modulus of 30.12 mag for NGC 3379 and of 31.26 mag for NGC 4489 (see discussion in next section). Identified foreground stars were not included in the fit.

$P_{\text {es }}$ was then calculated as the sum of:

$$
\begin{array}{r}
\sigma_{\mathrm{gc}}^{2}=\frac{1}{2} N_{\mathrm{ogc}} 10^{0.8\left[m_{1}-m_{\text {peak }}+0.4 \sigma^{2} \ln (10)\right]} \\
\operatorname{erfc}\left[\frac{m_{\mathrm{cut}}-m_{\text {peak }}+0.8 \sigma^{2} \ln (10)}{\sqrt{2} \sigma}\right]
\end{array}
$$

and

$\sigma_{\mathrm{bg}}^{2}=\frac{N_{\mathrm{obg}}}{(0.8-\gamma) \ln (10)} 10^{0.8\left(m_{1}-m_{\mathrm{cut}}\right)+\gamma\left(m_{\mathrm{cut}}\right)}$.

where $m_{1}$ is the zero magnitude which corresponds to a flux of $1 \mathrm{ADUs}^{-1}$. 
Finally, the apparent SBF magnitude was computed as:

$\bar{m}_{K}=-2.5 \log \left(P_{0}-P_{\mathrm{es}}\right)+m_{1}-\epsilon_{\mathrm{ext}} \sec (z)$

where $\epsilon_{\text {ext }}$ is the extinction coefficient, and $\sec (z)$ the airmass for the observations. Color term and redshift corrections are negligible (Liu et al. 2000). No galactic foreground reddening corrections were applied given that $E(B-V)=0.028$ for NGC 4489 and $E(B-V)=0.024$ for NGC 3379, implying $A_{K}=0.01$ for NGC 4489 and 0.009 for NGC 3379 (Schlegel et al. 1998). We measured $\epsilon_{\text {ext }}=0.1$ and $m_{1}=21.21 \pm 0.03 \mathrm{mag}$ for the Kitt Peak data and $\epsilon_{\text {ext }}=0.07$ and $m_{1}=22.18 \pm 0.03$ mag for the NTT data. The error on $\bar{m}_{K}$ is given as the standard deviation of the different annuli considered plus the entire field. To this error, the errors due to the zero point magnitude and $\epsilon_{\text {ext }}$ calibration have been added in quadrature. When a distance estimation from $\bar{m}_{K}$ is made, the uncertainty of the SBF absolute magnitude calibration $\bar{M}_{K}$ calculated from previous $K$-band SBF observations (Jensen et al. 1998) is also added in quadrature.

\section{Results}

\subsection{NGC 3379}

The resultant NGC 3379 power spectra derived from the NTT data are shown in Fig. 3. The results for each annulus for NTT data are listed in Table 1. The standard deviation from each annulus calculated as the variance among different low wavelength cuts is listed. To the fitting error, the errors due the photometric zero point calibration and to external source residual contribution subtraction were added in quadrature. The Kitt Peak data for NGC 3379 were obtained under non-photometric conditions and are excluded from the final analysis. In both NGC 3379 datasets, there is a tendency to have fainter fluctuations in the center of the galaxy (Sodeman \& Thomsen 1995).

From NTT data a value of $\bar{m}_{K}=24.72 \pm 0.06 \mathrm{mag}$ was derived. The error is given by adding in quadrature the standard deviation of the magnitudes derived in each annulus divided by the number of considered values. Pahre \& Mould (1994) derived a value of $\bar{m}_{K}=$ $24.48 \pm 0.1 \mathrm{mag}$ for NGC 3379 from the analysis of annuli between $\approx 4^{\prime \prime}$ and $\approx 45^{\prime \prime}$. They did not correct for contamination by external source contribution correction. This could explain why they reported slightly larger fluctuations. Based on the Tonry et al. (2000b) measurements of $\bar{m}_{I}=28.57 \pm 0.07 \mathrm{mag}$ and the most recent $(V-I)=1.193 \pm 0.015 \mathrm{mag}$ (Ferrarese et al. 2000; Tonry et al. 2000b) and the Tonry et al. (2000a) calibration:

$\bar{M}_{I}=(-1.74 \pm 0.08)+(4.5 \pm 0.25)((V-I)-1.15)$,

a distance modulus of $\left(\bar{m}_{I}-\bar{M}_{I}\right)=30.12 \pm 0.11 \mathrm{mag}$ was adopted.

Using the adopted $I$-band distance modulus, an absolute magnitude of $\bar{M}_{K}=-5.40 \pm 0.13 \mathrm{mag}$ was derived.
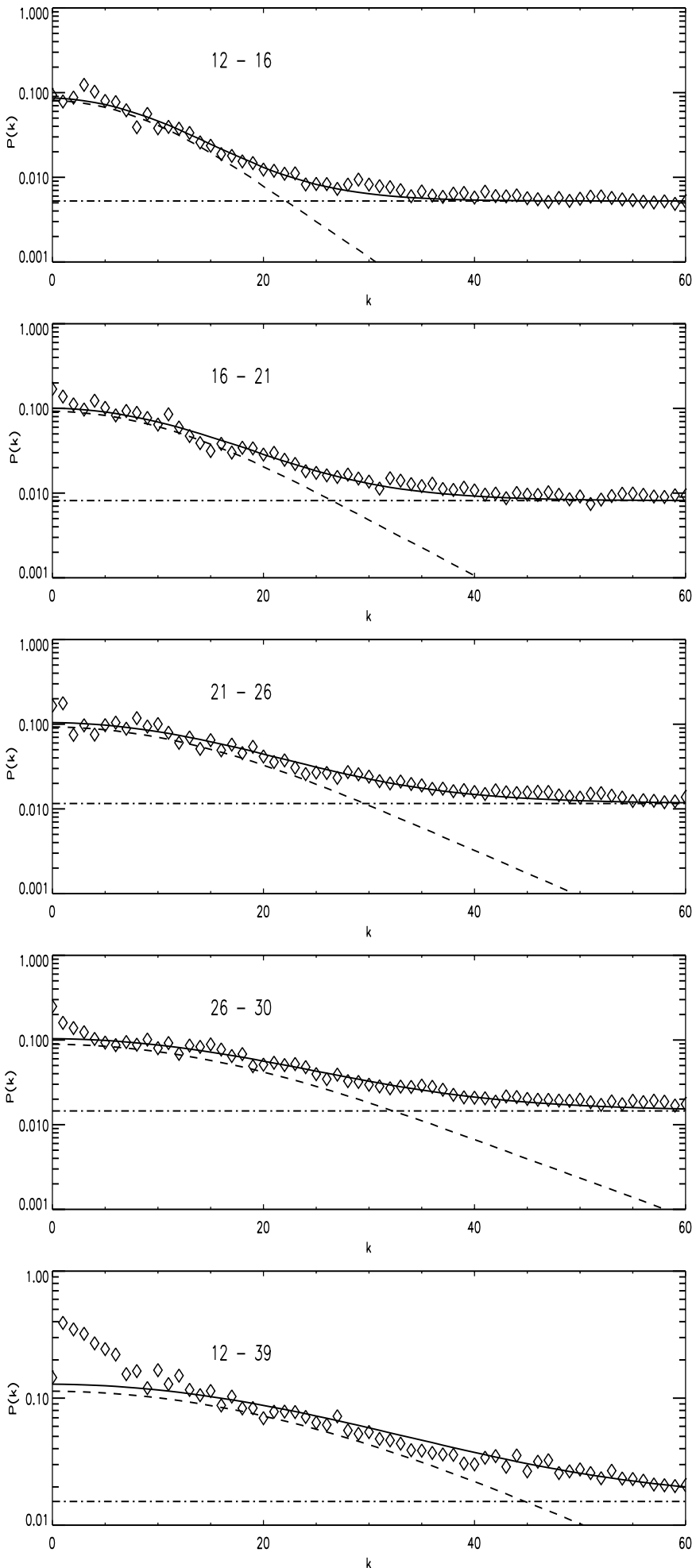

Fig. 3. NGC 3379 NTT power spectra. The NGC 3379 power spectra as a function of the wavenumber $k$ are shown for different annuli. The fit of the power spectrum is given by the continuous line, the PSF power spectrum by the dotted line and the dashed line is the fitted constant white noise spectrum. See Table 1

This value is consistent with measurements for other ellipticals presented in Jensen et al. (1998) sample and with 

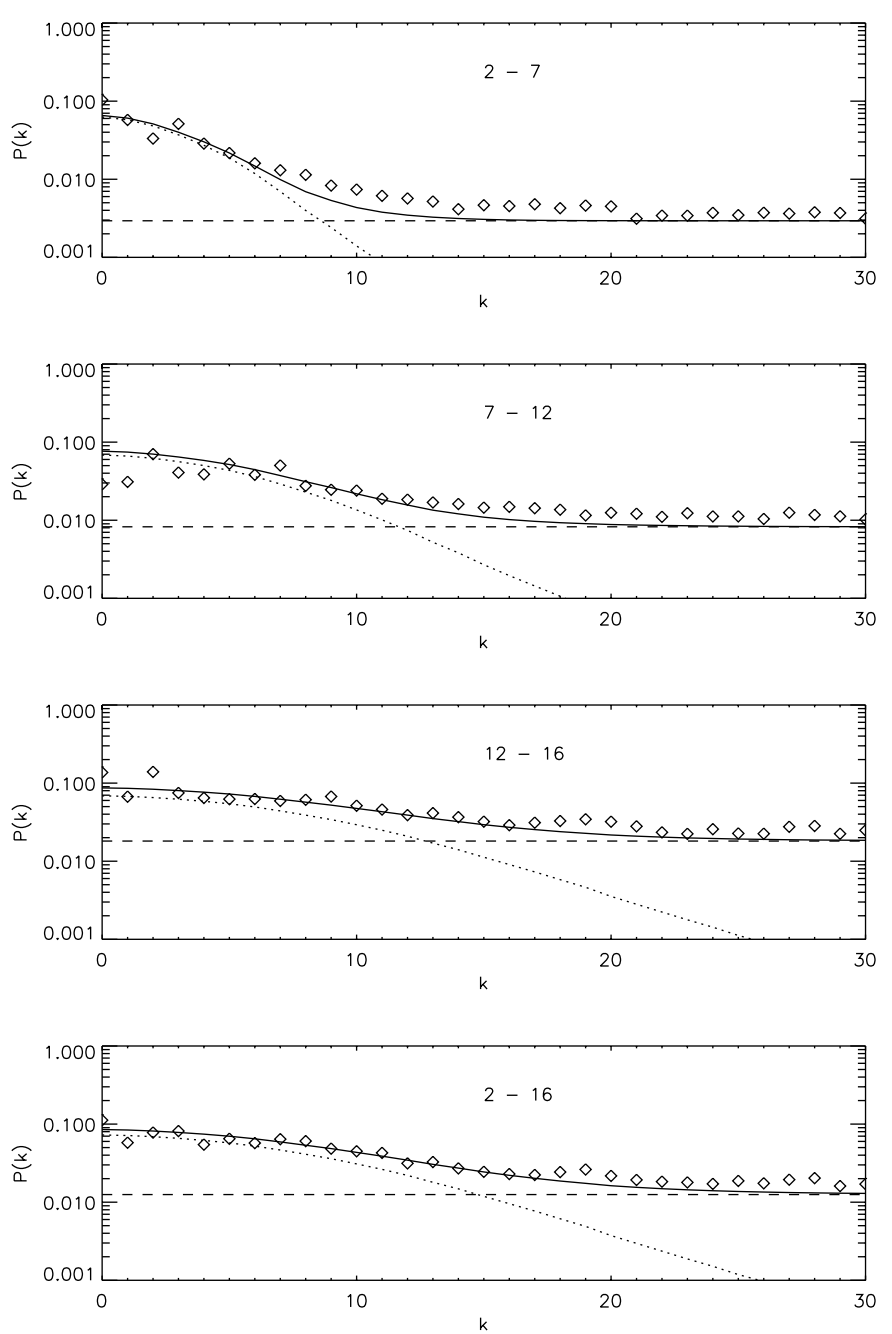

Fig. 4. NGC 4489 NTT power spectra. The NGC 4489 power spectra are shown as a function of the wavenumber $k$ from NTT observation in various annuli. The fit of the power spectrum is given by the continuous line, the PSF power spectrum by the dotted line and the dashed line is the fitted constant white noise spectrum. See Table 3

nearly solar metallicity, 15 Gyr old models from Bruzual \& Charlot (2000), as analyzed by Mei et al. (1999, 2000) and Liu et al. (2000) and from Worthey, as analyzed by Jensen et al. (1998).

\subsection{NGC 4489}

The power spectra for NGC 4489 as derived from the NTT data are shown in Fig. 4. The KPNO data produce similar results, albeit with lower signal-to-noise. External source detections from the NTT data were used to mask the external sources from the KPNO dataset after appropriate shifting and scaling. The results for each annulus for the respective datasets are listed in Tables 2 and 3 . The standard deviation from each annulus calculated as the variance among different low wavelength cuts is listed. To the fitting error the errors due to the photometric zero point calibration and to external source residual contribution subtraction are added in quadrature.
The signal-to-noise ratio $P_{0} / P_{1}$ for NTT data in the four annuli permit us to assess the SBF absolute magnitude for this galaxy with a percent error of approximately 10\% (Mei et al. 2000). From the NTT data we derive a value of $\bar{m}_{K}=24.97 \pm 0.06 \mathrm{mag}$ and from the KPNO data $\bar{m}_{K}=24.98 \pm 0.06 \mathrm{mag}$. The errors were calculated by adding in quadrature the standard deviation of the magnitudes derived in each annulus, divided by the number of considered values. Based on the most recent $\bar{m}_{I}=29.05 \pm 0.13 \mathrm{mag}$ and $(V-I)=1.046 \pm$ $0.015 \mathrm{mag}$ (Ferrarese et al. 2000; Tonry et al. 2000b), and using the $\bar{M}_{I}$ vs. $(V-I)$ Tonry calibration from Tonry et al. (2000a), a distance modulus for this galaxy equal to $\left(\bar{m}_{I}-\bar{M}_{I}\right)=31.26 \pm 0.13 \mathrm{mag}$ was adopted. $K$-band SBF absolute magnitudes of $\bar{M}_{K}^{\text {NTT }}=-6.29 \pm 0.14 \mathrm{mag}$ and $\bar{M}_{K}^{\mathrm{KPNO}}=-6.28 \pm 0.14 \mathrm{mag}$ were determined. The average of these two values is $\bar{M}_{K}=-6.29 \pm 0.10 \mathrm{mag}$.

This result is in agreement with the Jensen et al. (1998) measurement of $\bar{m}_{K}=24.99 \pm 0.35$ mag. It is not comparable with previous measurements by Pahre \& Mould (1994) and Jensen et al. (1996), because of the different treatment of the external source contribution to the fluctuations between this study and the previous studies.

The observed $\bar{M}_{K}$ is inconsistent with the mean empirical value derived by Jensen et al. (1998) $(-5.61 \pm$ $0.12 \mathrm{mag}$ ) at the two-sigma level, in the sense that NGC 4489 has brighter $K$-band surface brightness fluctuations than seen in bright, nearby, cluster ellipticals. This would appear to confirm the suggestion by Pahre \& Mould (1994) and Jensen et al. (1996) that NGC 4489 contains bright stellar component above the tip of the first ascent giant branch, a so-called extended giant branch population. Those initial studies argued that such a population could be similar to the $K$-band bright stellar population reported by Freedman (1992) and Elston \& Silva (1992) in M 32 (see also Luppino \& Tonry 1993; Davidge 2000). The exact nature of such extended giant branches remains controversial, with most studies arguing for the presence of an intermediate-age ( $\sim 2$ Gyr) AGB. The most likely alternative population currently appears to be high-metallicity first ascent giant stars, brighter than normally observed in Galactic globular clusters (Guarnieri et al. 1997).

However, NGC 4489 is in fact significantly fainter and bluer $(V-I=1.046)$ than the mean elliptical in the Jensen et al. calibration. This suggests that a comparison between the $\bar{M}_{K}$ measured for NGC 4489 and the Jensen et al. mean value may not be strictly valid. Since there are no published empirical $\bar{M}_{K}$ measures for ellipticals for $(V-I) \sim 1$, only theoretical predictions can be used to probe this color range. Unfortunately, currently available theoretical calculations of $\bar{M}_{K}$ provide conflicting results. Models based on the latest Bruzual \& Charlot models (e.g. Liu et al. 2000; Mei et al. 2000), predict $\bar{M}_{K}=-4.7$ to $-5.5 \mathrm{mag}$ for galaxies with $(V-I)$ color similar to NGC 4489 for single-burst old populations. For these models, the near-IR SBF magnitudes become brighter as the galaxies becomes redder. On the other hand, independent 
models by Blakeslee et al. (2000) predict $\bar{M}_{K}$ as bright as -6.5 in this $(V-I)$ color range, with near-IR SBF magnitudes becoming somewhat fainter as galaxies become redder.

In the end, one thing is certain: NGC 4489 illustrates that the Jensen et al. empirical mean $\bar{M}_{K}$ is not universal. Whether or not this means that NGC 4489 has an unusual stellar population mixture cannot be answered without further empirical observations of ellipticals with similar colors.

\section{Summary and conclusions}

New, high signal-to-noise $K$-band SBF measurements have been obtained for NGC 3379 and NGC 4489. In summary:

- $K$-band $\operatorname{SBF} \bar{M}_{K}$ values of $-5.40 \pm 0.13 \mathrm{mag}$ for NGC 3379 and $-6.29 \pm 0.10 \mathrm{mag}$ for NGC 4489 have been derived. Although the new apparent SBF magnitudes presented here are independent observations and are more accurate for NGC 4489 than previous determinations, the final $K$-band SBF absolute magnitudes rely on the accuracy of the respective distance moduli determined in the $I$-band;

- Keeping in mind the caveat above about absolute magnitudes, the measurement by Jensen et al. (1998) and Pahre \& Mould (1994) of large $K$-band SBF in NGC 4489 has been confirmed. These fluctuations could be the signature of an extended giant branch, but this cannot be confirmed until $K$-band SBF magnitudes are determined for a larger sample of galaxies similar in color to NGC 4489.

These results raise questions concerning the accuracy of $K$-band SBF distance measurements for elliptical galaxies. SBF measurements have been extended to this band in the belief that one could determine with the same accuracy $K$-band SBF distances to larger distances than possible at optical wavelengths. This is possible only if the contribution of external sources to the observed image fluctuations can be correctly estimated and if the $K$-band SBF absolute magnitude can be precisely calibrated. The result of this paper concerns the issue of calibration. The presently small sample of $K$-band measurements (around 20 ) would seem to suggest that $\bar{M}_{K}$ varies very little from galaxy to galaxy. However, our result - a galaxy with fluctuations different than the current average, illustrates the importance of an extended $K$-band calibration over a larger range of luminosity and color. Only larger samples will allow us to determine whether or not NGC 4489 (or for that matter M 32) is an exceptional case, and to understand the real potential of $K$-band SBF distance determinations.

Note added in proof: When this paper was refereed, we used galaxy colors and distance moduli from Ferrarese et al. (2000). When using these colors and distance moduli, we obtained absolute $K$-band magnitudes of $-5.32 \pm 0.13 \mathrm{mag}$ for NGC 3379 and $-6.18 \pm 0.09 \mathrm{mag}$ for NGC 4489. After our paper was accepted, Tonry et al. (2000b) published revised colors and distance moduli for NGC 3379 and NGC 4489 (among others). We have used the Tonry et al. revisions to update our $K$-band SBF magnitudes in the published version of our paper. The revised values are $-5.40 \pm 0.13 \mathrm{mag}$ and $-6.29 \pm 0.10 \mathrm{mag}$ for NGC 3379 and NGC 4489, respectively. These updated $K$-band SBF magnitudes do not change the conclusions of our paper. We thank John Blakeslee for bringing the Tonry et al. revisions to our attention.

Acknowledgements. S. Mei is grateful to P. Rosati and M. Romaniello for the useful discussions and acknowledges support from the European Southern Observatory Studentship programme and Director General's Discretionary Fund.

\section{References}

Ajhar, E. A., Lauer, T. R., Tonry, J. L., et al. 1997, AJ, 114, 626 Bertin, E., \& Arnouts, S. 1996, A\&AS, 117, 393

Blakeslee, J. P., Ajhar, E. A., \& Tonry, J. L. 1999, in PostHipparcos Cosmic Candles, ed. A. Heck, \& F. Caputo (Boston: Kluwer), 181

Blakeslee, J. P., \& Tonry, J. L. 1995, ApJ, 442, 579

Blakeslee, J. P., Vazdekis, A., \& Ajhar, E. A. 2000, MNRAS, in press [astro-ph/0008218]

Cowie, L. L., Gardner, J. P., Hu, E. M., et al. 1994, ApJ, 434,114

Davidge, T. J. 2000, PASP, 112, 1177

Elston, R., \& Silva, D. R. 1992, AJ, 104, 1360

Ferrarese, L., Ford, H. C., Huchra, J., et al. 2000, ApJS, 128,431

Freedman, W. L. 1992, AJ, 104, 1349

Frogel, J. A., Persson, S. E., Matthews, K., et al. 1978, ApJ, 220,75

Gebhardt, K., \& Kissler-Patig, M. 2000, AJ, 118, 1526

Guarnieri, M. D., Renzini, A., \& Ortolani, S. 1997, ApJL, 477, 21

Jensen, J. B., Luppino, G. A., \& Tonry, J. L. 1996, ApJ, 468,519

Jensen, J. B., Tonry, J. L., \& Luppino, G. A. 1998, ApJ, 505,111

Jensen, J. B., Tonry, J. L., \& Luppino, G. A. 1999, ApJ, 510, 71

Kissler-Patig, M., private communication

Lauer, T. R., Tonry, J. L., Postman, M., et al. 1998, ApJ, 499,577

Liu, M. C., Charlot, S., \& Graham, G. R. 2000, ApJ, accepted [astro-ph/0004367]

Luppino, G. A., \& Tonry, J. L. 1993, ApJ, 410, 81

Mei, S., Silva, D. R., \& Quinn, P. J. 1999, in Harmonizing Cosmic Distance Scales in a Post-HIPPARCOS Era, ASP, Conf. Ser. 167, ed. D. Egret, \& A. Heck, 279

Mei, S., Quinn, P. J., \& Silva, D. R. 2000, submitted to A\&A Pahre, M. A., \& Mould, J. R. 1994, ApJ, 433, 567

Pahre, M. A., Mould, J. R., Dressler, A., et al. 1999, ApJ, 515,79

Persson, S. E., Murphy, D. C., Krzeminski, W., et al. 1998, AJ, 116,2475 
Press, W. H., Flannery, B. P., Teukolsky, S. A., et al. Tonry, J. L., \& Schneider, D. P. 1988, AJ, 96, 807 1992, Numerical Recipes, (Cambridge University Press, Tonry, J. L., Ajhar, E. A., \& Luppino, G. A. 1990, AJ, New York)

Schlegel, D. J., Finkbeiner, D. P., \& Davis, M. 1998, ApJ, Tonry, J. L., Blakeslee, J. P., Ajhar, E. A., et al. 1997, ApJ, 500,525

Silva, D. R., \& Bothun, G. D. 1998, AJ, 116, 85

Sodemann, M., \& Thomsen, B. 1996, AJ, 111, 208

Sodemann, M., \& Thomsen, B. 1995, AJ, 110, 179

Thomsen, B. Baum, William A., Hammergren, M., et al. 1997, ApJ, 483, L37 475,399

Tonry, J. L., Blakeslee, J. P., Ajhar, E. A., et al. 2000a, ApJ, 530,625

Tonry, J. L., Dressler, A., Blakeslee, J. P., et al. 2000b, ApJ, in press [astro-ph/0011223]

Wainscoat, R., \& Cowie, L. 1992, AJ, 101, 332 